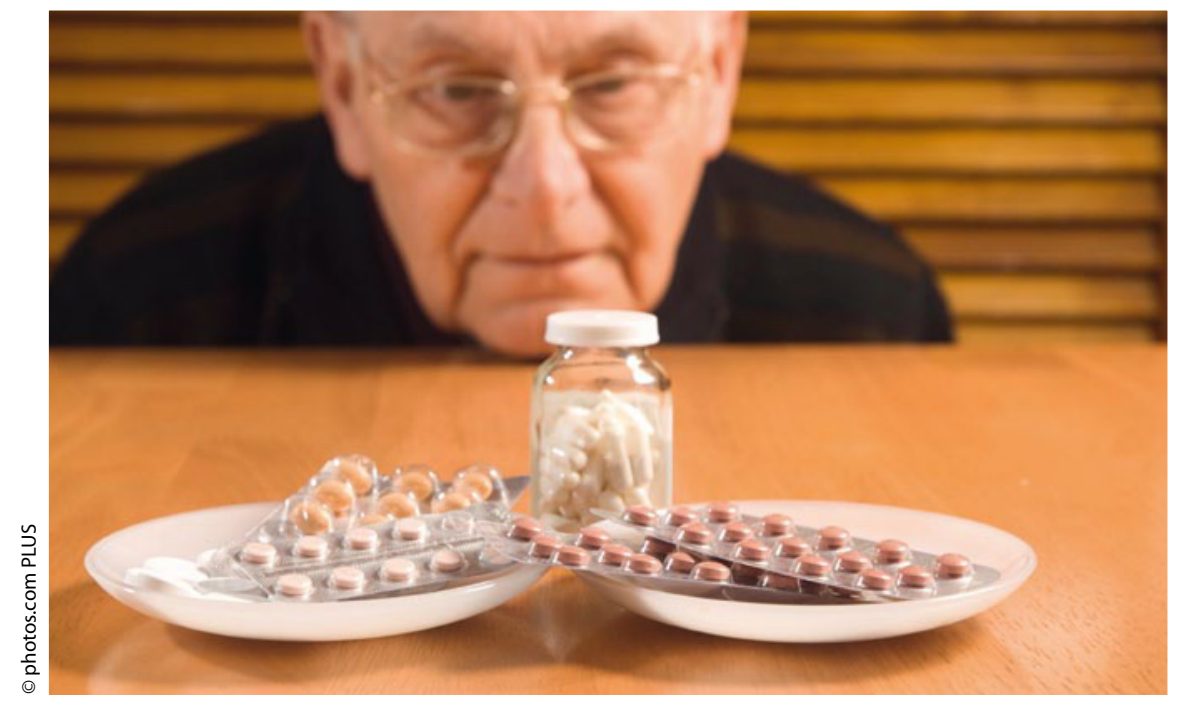

\title{
Multimorbide Patienten: Ist weniger Pharmazie mehr?
}

In einer prospektiven Studie aus Israel wurden 70 multimorbide Patienten (Durchschnittsalter 83 Jahre) untersucht, die im Mittel acht verschiedene Medikamente einnahmen. Die Indikation für diese Polypharmakotherapie wurde nach einem speziellen Schema kritisch geprüft.

- So wurden beispielsweise Nitrate als nicht indiziert erachtet, wenn sechs Monate keine Angina pectoris, Magensäurehemmer, wenn ein Jahr keine GIBlutung aufgetreten war. 311 Arzneien bei 64 Patienten wurden in Frage gestellt. Nach Rücksprache mit Patienten und Hausärzten wurden schließlich 256 Medikamente versuchsweise abgesetzt und die Patienten 19 Monate lang nachbeobachtet.

Lediglich sechs der 256 abgesetzten Medikamente mussten im Studienverlauf wieder angesetzt werden. Fast alle Nitrate, Benzodiazepine und Sulfonylharnstoffe erwiesen sich als verzichtbar, ebenso die Hälfte aller Antihypertensiva, Säureblocker und Statine und ein Drittel der Psychopharmaka. Keinem der Patienten ging es nach eigenem Empfinden schlechter, fast $90 \%$ berichteten sogar von einer Besserung ihres
Befindens. Bei 56 Patienten (88\%) kam es zu einer (teils drastischen) Besserung ihrer kognitiven Leistungsfähigkeit.

Die Autoren kommen zu dem Schluss, dass bei alten Menschen mit umfangreichen Medikamentenlisten bis zu 50\% der Arzneimittel abgesetzt werden können ohne Verschlechterung, in vielen Fällen sogar mit deutlicher Besserung ihres Gesundheitszustandes.

\section{Kommentar}

Polypharmazie, lange Medikamentenlisten, bis 20 Tabletten pro Tag bei hochbetagten gebrechlichen Patienten: Es ist leicht, darüber zu lamentieren. Es ist aber immer wieder schwer, Ordnung und ein sinnvolles Maß in jede einzelne dieser - oft im wahrsten Wortsinn schwindelerregenden - Listen zu bringen.

Das Schema, nach dem in der vorliegenden Studie die Medikamente reduziert wurden, nennt sich "good palliative geriatric practice". Mancheiner mag "palliativ" in Verbindung bringen mit "nichts mehr tun können". Das wäre jedoch falsch.

In einem kürzlich im British Medical Journal erschienenen Schwerpunkt zum Thema Palliativmedizin bei Alterskrankheiten habe ich eine Antwort gelesen auf die Frage: „A $\mathrm{Ab}$
Braucht er das

alles wirklich? wann ist man ein Palliativ-Patient?", die mir gut gefallen hat. Als Palliativ-Patienten gelten demnach betagte Patienten, deren Ableben innerhalb der nächsten 6-12 Monaten nicht überraschend sei, Patienten, die in ihren Alltagsaktivitäten eingeschränkt sind, die in den letzten sechs Monaten mehrfach im Krankenhaus waren oder bei denen eine NYHA III-IV Symptomatik oder eine höhergradige Demenz vorliegt. In diesen Fällen sollte man das Therapieziel strikt auf Symptomlinderung hin ausrichten.

Auch nach meiner Erfahrung zählen Nitrate, Säureblocker, NSAR, Statine, Neuroleptika, Allopurinol, Sulfonylharnstoffe und zahlreiche weitere Medikamente zu den Arzneien, die man oft ungestraft weglassen kann. Auch ein zu scharf ( $<140 / 85 \mathrm{mmHg}$ ) eingestellter Blutdruck schadet in der Regel mehr als er nützt. Dass in der vorliegenden Studie jedes zweite Medikament weggelassen werden konnte und es den Patienten dabei in puncto Lebensqualität und kog-nitiver Leistungsfähigkeit nicht nur nicht schlechter, sondern in $88 \%$ ",besser" und in zwei Drittel sogar "deutlich besser" ging, ist sehr eindrucksvoll. Bevor man nun definitiv folgern kann, gebrechliche Hochbetagte nähmen die Hälfte ihrer Medikamente ohne nützlichen oder sogar mit schädlichem Effekt, bedarf es natürlich einer größeren Studie mit einer Kontrollgruppe. Aber selbst wenn es eine solche Studie nie geben sollte (z. B. weil sich kein Sponsor findet), dürfen wir bereits heute bei der Pharmakotherapie gebrechlicher und hochbetagter Patienten den Fuß spürbar vom Gas nehmen. Das ",weniger ist mehr" ist wissenschaftlich belegbar.

J. ZEEH -

Für Interessierte stellen wir gerne unsere klinikinternen Leitlinien zur Medikamentenreduktion zur Verfügung (Mail an j.zeeh@sozialwerk-meiningen.de). Kritik und Rückmeldungen erwünscht.

\section{- D. Garfinkel, D. Mangin}

Feasibility study of a systematic approach for discontinuation of multiple medications in older adults. Arch. Int. Med. 170 (2010) 1648-1654 